\title{
ORIGINAL ARTICLE \\ Depressive mood in adults with spinal cord injury as they transition from an inpatient to a community setting: secondary analyses from a clinical trial
}

\author{
A Craig, R Guest, Y Tran and J Middleton
}

\begin{abstract}
Study design: Prospective cohort controlled trial design.
Objectives: (i) To investigate mood benefits of adding group cognitive behaviour therapy (group-CBT) to standard spinal cord injury (SCI) inpatient rehabilitation (SR) that included access to antidepressant medication and individually delivered CBT on demand. (ii) To determine whether those with elevated depressive mood during inpatient rehabilitation significantly improve.

Setting: $\mathrm{SCl}$ rehabilitation and community settings in New South Wales, Australia.

Methods: Participants included 50 adults with $\mathrm{SCl}$ who completed SCl rehabilitation that included group-CBT compared with 38 participants who also completed $\mathrm{SCl}$ rehabilitation that did not contain group-CBT. Comprehensive assessment occurred after admission, within 2 weeks of discharge and 12 months post-injury. Multivariate repeated measures analyses were conducted to examine differences between groups and over time.

Results: The addition of group-CBT to SR did not result in significant improvement in mood. However, participants with clinically elevated depressive mood assessed during inpatient rehabilitation experienced significant reductions in depressive mood when assessed in the community regardless of CBT dosage. Anxiety correlated with mood while no sociodemographic/injury factors correlated with mood at any time period except education level.

Conclusion: There were no mood advantages over time of adding group-CBT to inpatient SCl rehabilitation that contains individually delivered CBT on demand and access to antidepressant medication. However, findings showed those with elevated depressive mood during inpatient rehabilitation significantly improved when assessed in the community; however, their levels of depressive mood remain high. Future research should investigate the efficacy of providing individual preferences for managing depression in people with SCI. Spinal Cord (2017) 55, 926-934; doi:10.1038/sc.2017.41; published online 2 May 2017
\end{abstract}

\section{INTRODUCTION}

Spinal cord injury (SCI) is a neurological disorder occurring when the cord is severely bruised, lacerated, or severed due to traumatic injury or disease. ${ }^{1}$ In addition to loss of sensation, muscle paralysis and internal dysfunction, it can commonly lead to chronic pain, chronic fatigue and psychological morbidity..$^{2-11}$ The presence of psychological morbidity will more likely than not have a negative impact on adjustment to SCI. ${ }^{3,5-7}$ For example, depression has been associated with more severe physical complications in people with $\mathrm{SCI},{ }^{6,12}$ and risk of self-neglect and suicide is higher. ${ }^{13}$ It is also a concern that suicide rates following SCI have increased, whereas treatable causes of death such as septicaemia and pneumonia have significantly reduced since $1980 .{ }^{13}$ In addition, the transition to the community from inpatient rehabilitation involves new and ongoing stressors and demands that will challenge mood, including coping with medical complications, managing pain, maintaining healthy self-care, dealing with financial difficulties, lowering barriers to social participation and solving logistical difficulties with transportation. ${ }^{14}$

Depression is an episodic and often recurrent disorder. ${ }^{5-7,9}$ Therefore, in studies that estimate the rate of depressive mood in people following an SCI, it should be appreciated that the onset of the first episode of depression may predate the injury given that a history of premorbid psychological treatment has been shown to be a significant predictor of depression after sustaining an SCI. ${ }^{6}$ A systematic review of 18 studies that investigated rates of depressive mood following SCI, concluded that around 30\% of people with SCI had probable depression in the short- and long-term. ${ }^{15}$ A recent meta-analysis concluded rates of probable depression ranged between 19 and $26 \%$, with a mean prevalence estimate of $22 \%{ }^{11}$ Early prospective research by Kishi et al. ${ }^{16}$ found high rates of mood disorder of around $30 \%$ in the inpatient stage, declining to around $15 \%$ at a 6 months follow-up. Another early study found similar high rates of depressive mood in the inpatient stage; however, rates remained high 6 months to 2 years following discharge from hospital. ${ }^{17}$ Prospective research that employed structured psychiatric interviews found the rate of major depressive disorders (including bipolar disorders) to lie between 15 and 18\% 12 months post-injury, with an upper $95 \%$ confidence interval of $26 \% .{ }^{6}$ Alcohol abuse and suicidality were highly co-morbid with depressive disorders. ${ }^{6}$ In summary, it is likely that between 15 and $30 \%$ of

John Walsh Centre for Rehabilitation Research, Sydney Medical School-Northern, The University of Sydney, St Leonards, NSW, Australia

Correspondence: Professor A Craig, John Walsh Centre for Rehabilitation Research, Sydney Medical School-Northern, Kolling Institute of Medical Research, The University of Sydney, Corner Reserve Road \& First Avenue Royal North Shore Hospital, St Leonards, NSW 2065, Australia.

E-mail: a.craig@sydney.edu.au

Received 18 September 2016; revised 16 March 2017; accepted 21 March 2017; published online 2 May 2017 
people with SCI will exhibit clinically elevated depressive mood during inpatient rehabilitation and following transition into the community. This rate is much higher than the rate of mood disorders in the Australian community, ${ }^{18}$ and there is no compelling reason to suspect this differs internationally.

Latent class growth analysis research has provided rich information on trajectories of mood following SCI. Bonanno et al. ${ }^{19}$ followed a large SCI sample over 2 years, and found the majority had stable mood over the 2-year period and these were labelled resilient (66.1\%), a small percentage experienced improved mood (13.3\%), whereas $20.5 \%$ had a trajectory indicating the presence of depressive mood. Bombardier et al. ${ }^{20}$ examined mood pathways over a 12-month period following SCI. A majority (63.8\%) had an absence of depressive mood symptoms, a large minority (29.1\%) were experiencing persistent mild to moderate depression throughout the 12 months, while a small percentage $(7.1 \%)$ experienced chronic moderate to severe depressive mood. ${ }^{20}$ Guest et al. ${ }^{21}$ examined resilience pathways up to 12 months post-injury in adults with SCI. They found a majority had robust levels of resilience (61.3\%), while the remainder $(38.7 \%)$ had poor or deteriorating resilience. The three trajectory studies conducted with European, ${ }^{19}$ North American ${ }^{20}$ and Australian $^{21}$ participants produced similar proportions of those experiencing difficulties with mood or resilience.

SCI is currently managed using a multidisciplinary rehabilitation team approach consisting of pharmacological, medical, physical and psychosocial rehabilitation dimensions. ${ }^{22}$ Regrettably, controlled prospective research investigating the efficacy of SCI rehabilitation for reducing depressive mood is uncommon. For example, antidepressant medications (e.g. serotonergic and tricyclic medications) are commonly used to treat depression in people with $\mathrm{SCI},{ }^{7}$ but the benefit of these medications is uncertain..$^{23,24}$ However, a recent multisite double-blind randomized control trial showed that a 12-week trial with a flexible dose algorithm of the antidepressant venlafaxine XR resulted in improved mood compared with controls. ${ }^{23}$ Prospective controlled trials have provided preliminary evidence that cognitive behaviour therapy (CBT) integrated into multidisciplinary SCI rehabilitation leads to reduced depressive mood for inpatients with SCI, though methodological problems raise uncertainties about its generalizability. ${ }^{24-26}$ A recent meta-analysis concluded that adding CBT to SCI rehabilitation provides short-term benefits for reducing depressive mood, while long-term benefits are unsure. ${ }^{27}$ Prospective controlled studies in which CBT was delivered in the inpatient stage have found significant reductions in depressive mood in the short term, ${ }^{28,29}$ while others have found no improvements. ${ }^{30}$ A prospective inpatient study found significant reductions in depressive mood post-discharge only in those who had clinically elevated depressive mood during the inpatient phase, with continued improvements in mood continuing from 2 to 6 years following discharge. ${ }^{31,32}$ Further, CBT integrated into SCI rehabilitation has resulted in improved self-appraisal, ${ }^{8,26,29}$ while a recent controlled trial conducted by the authors found no benefit of providing group-CBT versus CBT delivered on demand to individuals in terms of resilience, for inpatients with SCI up to 12 months post-injury. ${ }^{21}$

Rates of depressive mood remain high as people transition into the community following SCI rehabilitation, ${ }^{6}$ even though inpatient rehabilitation has been shown to result in health and wellness benefits, ${ }^{33}$ and provides medical, physical and psychosocial interventions that typically include pharmacological treatment, physical and occupational therapies, counselling, CBT and introduction to assistive technology. ${ }^{22,34}$ Nevertheless, additional research is required to clarify optimal dosage and style of delivery of CBT integrated within SCI rehabilitation. For instance, is the addition of group sessions of CBT to standard rehabilitation (SR) already containing individual CBT on demand associated with additional improved mood? Further, is it those with clinically elevated depressive mood who are more likely to benefit from their inpatient rehabilitation? This paper presents secondary analyses from clinical trial data that investigated the impact of adding group-CBT sessions to standard inpatient SCI rehabilitation on levels of resilience. ${ }^{21}$ The secondary analyses presented in this paper involved the efficacy of improving mood by adding group-CBT sessions to standard inpatient SCI rehabilitation. Results are also presented on the benefits of SCI rehabilitation for those who have clinically elevated depressive mood. It was hypothesized that those receiving SR with group-CBT will have lower rates of depressive mood compared with those receiving SR. It was also hypothesized that those with elevated depressive mood in the inpatient stage will have significantly improved mood when assessed in the community.

\section{MATERIALS AND METHODS}

\section{Study participants}

Inclusion criteria involved: (i) English speaking; (ii) aged 18-80 years; (iii) admission to an SCI unit with an acute SCI for the first time as an inpatient, and (iv) adequate cognitive performance to complete the questionnaires. Exclusion criteria included (i) the presence of a severe traumatic brain injury, (ii) very incomplete or ambulant and (iii) severe cases of psychiatric disorder (e.g. schizophrenia or bipolar disorder with severe manic episodes). Full details concerning recruitment have been provided elsewhere. ${ }^{6,21}$ In summary, participants included 91 adults with acute SCI who met inclusion criteria. Participants were consecutively admitted to three adult SCI units in Sydney, Australia over a period of 33 months (April 2010-January 2013). Three withdrew from the study, resulting in 88 participants. Participants were aged between 18 and 80 years $(M=42.65$, s.d. $=17.85)$ with 62 males and 26 females.

A further 170 patients were also admitted to the three SCI units within the 33 months of the study who were not recruited. The majority $(n=110)$ failed to meet inclusion criteria for reasons such as having severe traumatic brain injury, being too old, being very incomplete or ambulant, and having a dementia, while 60 who met inclusion criteria declined to consent for reasons such as being unwell at the time or having conflicting rehabilitation schedules. These 60 were not significantly different to the 88 study participants for mean age, sex ratio or length of stay in the SCI Units.

Thirty-eight participants ( 34 males; 4 females; mean age $=41.60$ years; s.d. $=18$; minimum-maximum: $18-79$ years) entered the study in the first phase of the clinical trial (i.e. first 16 months) and were assigned to the SR control group. This group received SR in which all participants had access to interventions for depressive mood such as antidepressant medications and individual CBT on demand delivered by a resident clinical psychologist. For the second phase of the trial, 50 (28 males; 22 females) participants ( mean age $=43.49$ years; s.d. $=17.71 ; 18-80$ years) entered the study in the following 17 months and were assigned to the group-CBT intervention group, that is, participants received additional group-based CBT sessions within their rehabilitation, which also contained antidepressant pharmacological treatment and individual CBT on demand. Both groups received the same amount of hours of overall rehabilitation services. Figure 1 shows a CONSORT diagram with recruitment details and number of drop-outs in both groups. Table 1 shows sociodemographic and injury details for the entire sample and the two groups.

\section{Research ethics and trial registration}

Full compliance with the Code of Ethics of the World Medical Association occurred, and research ethics committee approval from the relevant institution was obtained. Written consent was obtained prior to entry in the study by staff in the SCI units. The Universal Trial Number is U1111-1134-5485, and the 


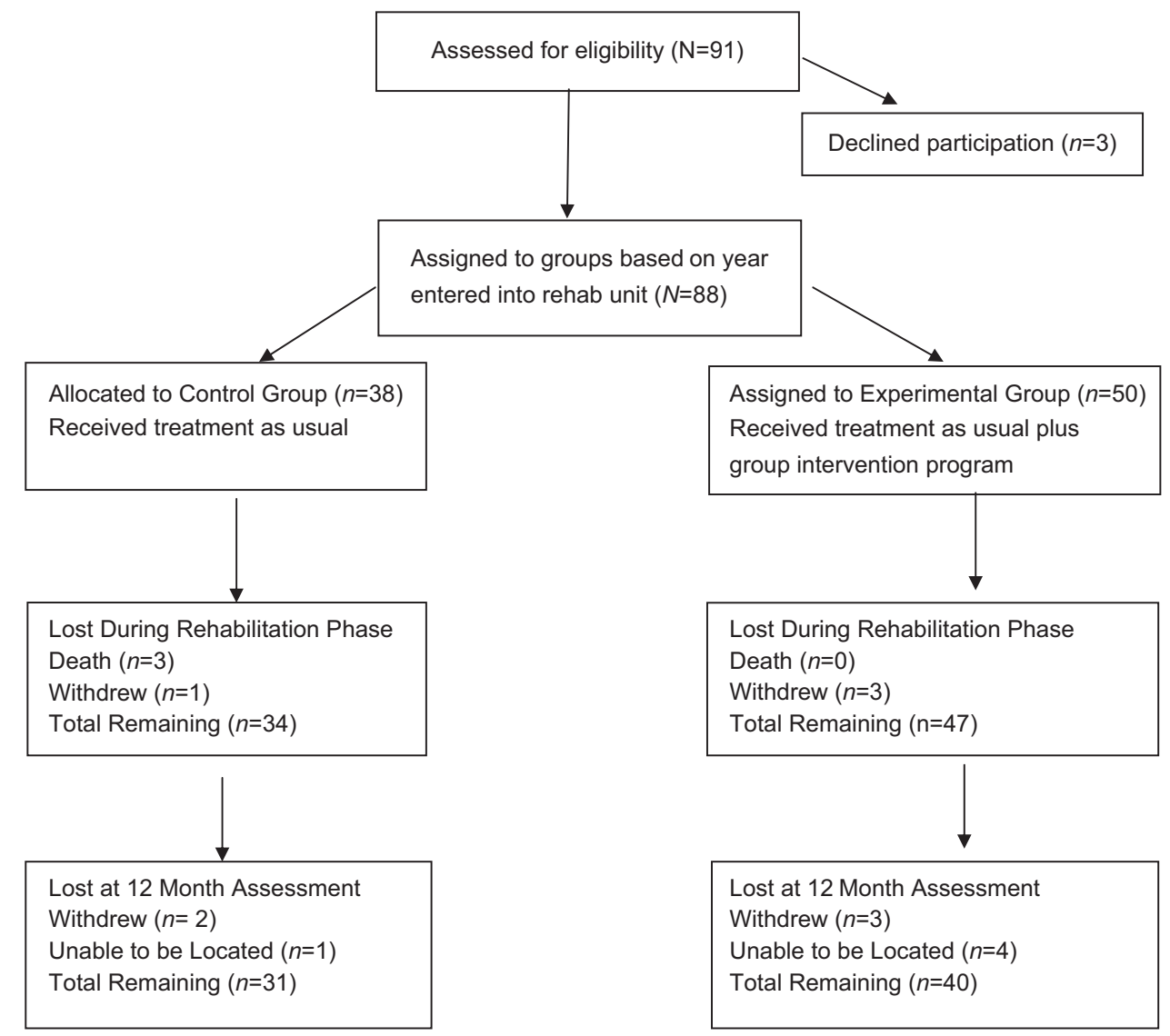

Figure 1 Consort diagram of recruitment and loss at each stage of assessment including decline to participate, consent to participate, loss due to death, unwillingness to continue with participation or unable to locate after 6 months of community living. Note, 12-month assessment is the 12-month post-injury assessment.

trial registration number is ACTRN12612000984897 (Australian \& New Zealand Clinical Trials Registry).

\section{Study design and procedure}

Recent guidelines have stressed the importance for social and psychological research to utilize randomized control trials to test the efficacy of psychological interventions. ${ }^{35,36}$ The investigators agreed that randomizing participants to groups would have been scientifically advantageous; however, it was also believed a randomized control trial would not have been appropriate for this study. This was due to: (i) the difficulty of blinding participants to a psychological therapy like CBT, (ii) the likely contamination between the control and intervention groups given the small numbers of inpatients in the three SCI Units in any one year would make randomization to a psychological intervention difficult. For example, New South Wales (NSW) has up to 100 new acute SCI admissions each year, ${ }^{37}$ and (iii) active communication transpires among the SCI population in NSW, decreasing chances that randomization would be effective. As a consequence, a quasiexperimental covariance design was chosen in which confounding variables suspected of independently influencing the outcome variable were included in the analyses as covariates. ${ }^{36}$

To determine the impact of the addition of group-CBT to SR, a prospective repeated measures group cohort design was employed. Groups were assessed on three occasions: the first assessment occurred within 1 month of admission to the SCI unit as an inpatient, the second, within 2 weeks of discharge from hospital and the third assessment was conducted in the community, mostly in the participant's home after 6 months of community living, which was at least 12 months post-injury. The 12 months post-injury included: (i) a mean of 7.3 weeks (s.d. $=6.1$; that is, from the time of the injury, through acute care to admission to the Rehabilitation Units when the initial assessment occurred); (ii) the mean length of stay in the SCI Units was 23.1 weeks (s.d.=9) and (iii) a mean 6 months or 26 weeks in the community. Assessments 1 and 2 were conducted face-to-face when an inpatient. Assessment 3 was conducted when living in the community, by face-to-face, or by Skype or telephone if face-to-face assessment was not possible. If the third assessment was conducted by Skype or telephone, the measures (except the psychiatric structured interview) were mailed to the participant. Participants were given breaks throughout the assessments to reduce fatigue occurring in the participants.

\section{Content of the group-CBT}

The group-CBT consisted of structured sessions designed to enhance mood in the participants through teaching cognitive and behavioural skills. Session content and structure were provided in a written manual for clinicians and participants. The group-CBT involved the addition of six 2-h weekly sessions so that each participant received up to $12 \mathrm{~h}$ of group-CBT. Sessions occurred in consecutive weeks, in groups of up to six participants, composed of males or females. Sessions involved psychological skill-based components intended to improve self-management of distress, including psycho-education, cognitive restructuring of unhelpful thinking, mindfulness, anxiety management skills such as breath rate control, visualization and problem solving skills. Participants were requested to complete homework on each session's content. Sessions were conducted by the SCI unit's clinical psychologist(s), who received training in delivery of the CBT protocol. Individual CBT sessions provided in SR and available to both groups were comprised of sessions personalized to an individual's problems and needs, whereas group-CBT sessions did not focus on specific individual problems and experiences, but more on primary skills and strategies.

To allow comparisons between the two groups on the effects of the additional hours of group-CBT, it was crucial that participants received 
Table 1 Demographic and injury characteristics for the SR Control $(n=38)$, group-CBT $(n=50)$ and total $\mathrm{SCl}$ participants $(N=88)$

\begin{tabular}{lrrr}
\hline Characteristic & Total & SR Control & Group-CBT \\
\hline Sex & & & \\
$\quad$ Female, $n(\%)$ & $26(29.55)$ & $4(10.53)$ & $22(44.0)$ \\
Males, $n(\%)$ & $62(70.45)$ & $34(89.47)$ & $28(56.0)$
\end{tabular}

Age

$$
\text { No. of years, M (s.d.) }
$$

$$
42.7 \text { (17.7) }
$$

$41.6(18.0)$

$43.5(17.7)$

Education

$$
\text { No. of years, M (s.d.) }
$$

$$
12.8(2.5)
$$

$12.6(2.5)$

$12.9(2.5)$

Cause of SCI, $n$ (\%)
MVA
Falls
Sport
Non-trauma
Shooting/assault
Other

$\begin{array}{ccc}26(29.88) & 14(36.84) & 12(24.49) \\ 27(31.06) & 13(34.21) & 14(28.57) \\ 10(11.49) & 4(10.54) & 6(12.24) \\ 10(11.49) & 3(7.89) & 7(14.29) \\ 2(2.29) & 1(2.63) & 1(2.04) \\ 12(13.79) & 3(7.89) & 9(18.37)\end{array}$

Employment status pre-injury, n (\%)

Employed
Pensioner
Unemployed
Student
Other

$\begin{array}{ccc}61(70.11) & 27(71.05) & 34(69.39) \\ 14(16.09) & 6(15.79) & 8(16.33) \\ 3(3.45) & 2(5.26) & 1(2.04) \\ 5(5.75) & 1(2.64) & 4(8.16) \\ 4(4.60) & 2(5.26) & 2(4.08)\end{array}$

$54(61.36) \quad 25(65.79) \quad 29(58.00)$

$34(38.64) \quad 13(34.21) \quad 21(42.00)$

Extent of impairment, $n(\%)$

Complete

Incomplete

$42(48.28)$

45 (51.72)

$20(52.63)$

$22(44.90)$

27 (55.10)

TBI

Yes

No

$18(20.45)$

8 (21.05)

$10(20.00)$

$70(79.55) \quad 30(78.95)$

40 (80.00)

\section{Cognitive capacity (NUCOG)}

Total, M (s.d.)

Norm, $n(\%)$

Below norm, $n(\%)$

Premorbid Psych Rx, n (\%)

Yes

No

$28(32.18) \quad 14(36.84) \quad 14(28.57)$

$59(67.82) \quad 24(63.16) \quad 35(71.43)$

Partner, $n$ (\%)

$\begin{array}{llll}\text { Yes } & 43(48.86) & 19(50.00) & 24(48.00) \\ \text { No } & 45(51.14) & 19(50.00) & 26(52.00)\end{array}$

Abbreviations: MVA, motor vehicle accident; Premorbid Psych Rx, premorbid psychological treatment; TBI, traumatic brain injury.

Note: For the total group for employment status and extent of impairment $n=87$, for the group-CBT, $n=49$ for cause of SCI, extent of impairment, employment and cognitive capacity screen (NUCOG), due to missing data in each of these variables.

a similar dosage of individual CBT sessions and similar proportion was prescribed antidepressant medication. This was the case. Rehabilitation in the SCI Units over the life of the study had minimal therapy changes. Like those in the SR group, group-CBT participants also had access to individual CBT on demand, that is, 1 -h non-focussed counselling sessions when requested by the participant or believed necessary by a member of the rehabilitation team. All participants received two $1-\mathrm{h}$ information sessions, first at admission to the rehabilitation unit and the second just before discharge from hospital. Access to patient records showed that the control (SR) and intervention group (group-CBT) received similar amounts of individual hourly psychological sessions, with a mean $8.3 \mathrm{~h}$ per person in the SR group and a mean $8.4 \mathrm{~h}$ per person receiving group-CBT. Also, 19.4\% of participants in the SR control (7/36) and $29.7 \%$ (14/47) of participants in the group-CBT were using antidepressant medication during the inpatient phase, and a $\chi^{2}$ test concluded this rate of use was not significantly different between groups $\left(\chi_{1}^{2}=1.42, P=0.24\right)$.

\section{Measures}

A psychologist independent of the delivery of the CBT group intervention conducted the assessment. Assessment at admission included sociodemographic factors such as sex, age, marital/partner status, years of education, weeks since the accident, cause of SCI, pre-injury employment status, level of injury (paraplegia versus tetraplegia), prior history of psychological/psychiatric treatment, compensation status, completeness of lesion, medications prescribed when an inpatient and whether they had received a co-morbid traumatic brain injury (from medical records). Psychotropic medications were also noted. Completeness of lesion was determined by medical specialists based on International Standards for Neurological Classification of SCI (http://ais.emsciorg/).

The Hospital Anxiety and Depression Scale (HADS) measures depressive mood and anxiety. Research has demonstrated the HADS to have appropriate psychometric properties and its items have been shown to be relatively free of SCI-related bias. ${ }^{38}$ A score $\geqslant 8$ on the HADS depressive mood domain indicates probable depression in adults with SCI, so this score was used to indicate the probable presence of clinically elevated depressive mood. ${ }^{38}$

Additional measures that had theoretical or historical links to depressive mood were also included in the assessments. Chronic fatigue was assessed by the Fatigue Severity Scale, shown to be a reliable and valid measure of fatigue, with high scores indicating increased fatigue. ${ }^{39}$ Participants self-rated their average pain intensity over the past week using a 0 to 10 numerical rating scale, with $0=$ 'no pain' and $10=$ 'pain as bad as can be imagined.' Numerical pain rating scales have acceptable test-retest reliability and validity. ${ }^{40}$ Pain catastrophizing was measured by a 9-item subscale of the Pain Related Self-Statements Scale (PRSS). ${ }^{41}$ High scores indicate escalated catastrophizing about their pain. The scale has acceptable reliability, validity and sensitivity. ${ }^{41}$ Cognitive capacity was assessed by the Neuropsychiatry Unit Cognitive Assessment Tool (NUCOG), a reliable and valid measure of cognitive impairment in people with neurological disorders. ${ }^{42}$ The NUCOG provides a total score of cognitive performance in addition to five domains including attention and memory. ${ }^{42}$ Functional capacity was measured by the Functional Independence Measure, where low scores indicate low functional capacity. ${ }^{43}$ The Functional Independence Measure has acceptable reliability and inter-rater agreement and includes self-care, grooming, mobility and sphincter control domains. ${ }^{43}$ The SCI Secondary Conditions Scale (SCS) was used to assess severity of secondary complications. ${ }^{44}$ It rates the severity of 16 health conditions including pressure ulcers, autonomic dysreflexia, bladder and bowel dysfunction, pulmonary health, circulatory problems, sexual dysfunction and chronic pain. Higher scores indicate increased severity of secondary complications. The SCS has acceptable validity and reliability. ${ }^{44}$

\section{Statistical methods}

The participant sample size of around 40 and 50 in each group provided 'a priori' statistical power of at least 70\% (assuming a low-medium effect size of 0.3 and an $\alpha$ of 0.05 , using a MANCOVA) to test the efficacy of adding group-CBT to SR. ${ }^{45}$ Previous research has shown CBT intervention effect sizes ranging between small $(0.2)$ for those with low depressive mood to moderate $(>0.50)$ for those with high depressive mood. ${ }^{25,28,31}$ General Linear Model repeated measures MANCOVA followed by Bonferroni $t$-tests were used to determine whether differences in depressive mood occurred over time as a function of the addition of group-CBT. To control for loss of cases through missing data, we used a regression substitution technique for up to $10 \%$ of 
cases. This preserved statistical power over time. The dependent variable for all three time periods was the HADS depressive mood domain score. Baseline variables found to be significantly different between the two groups were entered into the MANCOVA as covariates. In addition, in order to substantiate a claim of no difference between the CBT and SR groups, an equivalence test called the Two One-Sided Tests (TOST) based on Welch's $t$-test, was used to reject statistically the presence of differences large enough to be considered worthwhile (Web address for TOST: https://osf.io/preprints/psyarxiv/97gpc). ${ }^{46}$ To determine whether the two groups were equivalent, an upper and lower equivalence bound is specified based on the smallest effect size of interest. A HADS score of \pm 2 from the mean was chosen as the boundary for scores to be within a normal range for equivalence.

To determine whether participants with elevated depressive mood improved their mood from rehabilitation to the 12 months post-injury assessment, the entire sample of 88 was divided into two subgroups. The sample was split so that those with HADS depressive mood scores of $\geqslant 8$ taken in the baseline admission assessment were placed in the clinically elevated depressive mood subgroup, while the other subgroup had scores $<8$. Repeated measures MANOVA followed by Bonferroni $t$-tests were then used to determine differences. Dichotomizing a continuous variable like the HADS may reduce statistical power and cause under-utilization of data that falls close to the cutoff score. ${ }^{47}$ However, analyses conducted on the split sample demonstrated post hoc statistical power to range between 80 and $99 \%$, sufficient to protect against risk of type II errors. Eta-squared $\left(\eta^{2}\right)$ values are provided with the multivariate analyses as an indication of the size of the difference between the subgroups. An $\eta^{2}$ of around 0.03 is considered small, 0.13 is considered a medium difference and over 0.2 is considered a large and substantial difference. ${ }^{48}$ Post hoc statistical power for each analysis is also provided. Pearson or pointbiserial correlation coefficient analyses were conducted to calculate associations between depressive mood and sociodemographic, injury and psychological variables. Statistica version 12 (Statsoft, www.statsoft.com.au) was used to conduct statistical analyses.

\section{RESULTS}

Table 2 shows descriptive values for psychometric measures assessed for the group-CBT and SR groups as well as for the entire sample at admission, discharge and 12 months post-injury. Cognitive capacity and functional independence were assessed only during the rehabilitation phase. Inspection of Table 2 shows that the two groups had similar baseline scores for cognitive capacity, functional independence, fatigue, pain intensity and pain catastrophizing $(P>0.05)$. However, participants receiving the group-CBT had significantly higher admission anxiety and SCS scores compared with the SR controls $(P<0.05)$. Therefore, baseline HADS anxiety and SCS scores were entered into the repeated measures MANCOVA as covariates. Change in depressive mood in the two groups as participants transitioned from an inpatient rehabilitation setting into the community is shown in Table 2 and Figure 2. The MANCOVA indicated there were no significant differences in depressive mood between the two groups over time: Wilks $\lambda=0.99, \mathrm{~F}_{2,124}=0.04, P=0.96, \eta^{2}=0.001$, power $=5 \%$. The TOST test of equivalence found that depressive mood was equivalent between the two groups at admission $(P<0.05)$ and discharge $(P=0.01)$, and there was a trend to significance at 12 months post-injury $(P=0.076)$. Table 2 also shows pain intensity significantly increased for the entire group when assessed in the community: Wilks $\lambda=0.81, \mathrm{~F}_{2,94}=4.9, P<0.001, \eta^{2}=0.08$, power $=74 \%$; however, there were no differences in pain intensity increases over time between the group-CBT and SR control: Wilks $\lambda=0.97, \mathrm{~F}_{2,94}=0.61, P=0.55, \eta^{2}=0.01$, power $=15 \%$.

Table 3 shows clinically elevated rates of depressive mood (probable depression) in the group-CBT, SR control as well as for the total sample over the three time periods. Clinically elevated depressive mood for the total sample ranged between $24.1 \%$ at admission, $24.4 \%$ at discharge rising to $26.8 \% 12$ months post-injury (i.e. about 6 months after discharge). Inspection of Table 3 shows that the SR controls had lower rates of clinical depressive mood than group-CBT, though difference in rates had reduced by the 12-month post-injury assessment.

Figure 3 shows change in depressive mood over time in those with clinically elevated depressive mood versus those with normal levels of mood, irrespective of whether they were in the group-CBT or SR group in the prior analyses. Bonanno and colleagues ${ }^{19}$ isolated a resilient group with normal mood in their research, and therefore the normal mood group shown in Figure 3 has been labelled 'resilient'. Repeated measures MANOVA indicated significant reductions in depressive mood occurred over time: Wilks $\lambda=0.70, \mathrm{~F}_{2,138}=18.2$, $P<0.0001, \eta^{2}=0.21$, power $=99 \%$. Bonferroni $t$-tests indicated the resilient group did not change significantly over time, while depressive mood scores at admission and discharge in the clinically elevated group were significantly higher than mood at 12 months post-injury for both groups $(P<0.001)$. Table 4 shows HADS depressive mood scores for the resilient and probable depression groups over time. Table 5 shows antidepressant medication in the total sample at 6 months post-discharge as a function of clinically elevated depressive mood (HADS $\geqslant 8$ ). The overall percentage of participants prescribed antidepressant medications was $26.1 \% \quad(n=18 / 69)$. Surprisingly, only 4 of 18 of those with clinically elevated depressive mood at 12 months post-injury were taking antidepressants.

Table 6 shows correlation coefficients between the depressive mood score at the three time periods and the psychological factors for the total group. As expected, anxiety correlated highly with depressive mood across all time periods, while higher levels of fatigue were associated with higher depressive mood over the three time periods. Pain intensity was not significantly associated with depressive mood except at discharge. Higher levels of pain catastrophizing were associated with higher depressive mood at various time points, while higher secondary conditions scores were associated with higher depressive mood 6 months post-discharge. Few sociodemographic or injury factors correlated significantly with depressive mood at admission, discharge or 6 months after discharge over the total group. Years of education correlated negatively with depressive mood at the 6-month assessment $(r=-0.34, P<0.01)$, indicating a weak association exists between higher years of education and lower levels of depressive mood. The presence of a traumatic brain injury, premorbid psychological treatment and level of functional independence (Functional Independence Measure) were not significantly associated with depressive mood at any time period. Cognitive capacity (NUCOG total score) correlated significantly and negatively with depressive mood 6 months after discharge $(r=-0.31, P<0.05)$, indicating lower cognitive capacity was associated with higher depressive mood. Depressive mood at admission correlated significantly with mood at discharge and 6 months after discharge $(r=0.76, P<0.001$; $r=0.38, P<0.01$, respectively), while depressive mood at discharge correlated $r=0.41(P<0.01)$ with mood 6 months after discharge.

\section{DISCUSSION}

SCI rehabilitation is believed to be effective at helping people adjust following discharge. ${ }^{33}$ Nevertheless, rates of depression during rehabilitation and following discharge into the community remain substantially elevated. ${ }^{6,7,11,15-17}$ Based on prior work and data from this current research suggest that the occurrence of major depressive disorder would lie between 16 and $38 \%$ of the adult SCI population during rehabilitation and after they transition into the community. This rate is unacceptably high, being much higher than rates found in 
Table 2 Depressive mood, anxiety, fatigue, pain intensity, pain catastrophizing, cognitive capacity, functional independence and severity of secondary conditions at initial, discharge and 12-month post-injury assessments for the SR Control $(n=38)$, Group-CBT ( $n=50)$ and total group $(N=88)$

\begin{tabular}{|c|c|c|c|c|c|c|}
\hline \multirow[t]{2}{*}{ Variable } & \multicolumn{2}{|c|}{ SR Control } & \multicolumn{2}{|c|}{ Group-CBT } & \multicolumn{2}{|c|}{ Total } \\
\hline & $M(s . d)$. & $95 \% \mathrm{Cl}$ & $M(s . d)$. & $95 \% \mathrm{Cl}$ & $M(s . d)$. & $95 \% \mathrm{Cl}$ \\
\hline Initial $(n=88)$ & $5.08(3.12)$ & $4.05-6.10$ & $5.81(3.99)$ & $4.67-6.96$ & $5.50(3.64)$ & $4.72-6.27$ \\
\hline Discharge $(n=81)$ & $4.73(3.70)$ & $3.49-5.96$ & $4.93(3.66)$ & $3.83-6.03$ & $4.84(3.66)$ & $4.04-5.64$ \\
\hline 6 months $(n=71)$ & $4.93(3.62)$ & $3.55-6.31$ & $5.81(3.57)$ & $4.70-6.92$ & $5.45(3.60)$ & $4.60-6.30$ \\
\hline Initial $(n=88)$ & $4.45(4.02)$ & $3.13-5.77$ * & $5.89(4.01)$ & $4.75-7.05^{*}$ & $5.26(4.05)$ & $4.40-6.13$ \\
\hline Discharge $(n=81)$ & $4.43(3.98)$ & $3.10-5.76$ & $4.73(3.72)$ & $3.61-5.85$ & $4.60(3.82)$ & $3.76-5.44$ \\
\hline 6 Months $(n=71)$ & $5.41(4.48)$ & $3.71-7.12$ & $5.85(3.90)$ & $4.64-7.07$ & $5.68(4.12)$ & $4.70-6.65$ \\
\hline \multicolumn{7}{|l|}{ Fatigue (FSS) } \\
\hline Initial $(n=88)$ & $3.63(1.3)$ & $3.2-4.1$ & $3.81(1.5)$ & $3.4-4.2$ & $3.73(1.4)$ & $3.4-4.0$ \\
\hline \multicolumn{7}{|l|}{ Pain intensity } \\
\hline Initial $(n=88)$ & $3.57(3.1)$ & $2.5-4.6$ & $4.08(3.2)$ & $3.2-5.0$ & $3.85(3.1)$ & $3.2-4.5$ \\
\hline Discharge $(n=81)$ & 3.18 (2.6) & $2.3-4.1$ & $3.44(2.5)$ & $2.7-4.2$ & $3.34(2.5)$ & $2.8-3.9$ \\
\hline 6 Months $(n=71)$ & $4.72(2.3)$ & $3.6-5.9$ & $4.84(2.4)$ & $3.9-5.7$ & $4.80(2.4)$ & $4.1-5.5^{* *}$ \\
\hline \multicolumn{7}{|c|}{ Pain catastrophization (PRSS) } \\
\hline Initial $(n=86)$ & $1.01(1.0)$ & $0.65-1.4$ & $1.40(1.6)$ & $0.92-1.8$ & $1.22(1.4)$ & $0.92-1.5$ \\
\hline Discharge $(n=81)$ & $0.93(1.0)$ & $0.56-1.3$ & $0.91(0.9)$ & $0.63-1.2$ & $0.92(.96)$ & $0.70-1.1$ \\
\hline 6 Months $(n=71)$ & $0.95(1.1)$ & $0.55-1.4$ & $1.20(1.2)$ & $0.82-1.6$ & $1.10(1.1)$ & $0.83-1.4$ \\
\hline \multicolumn{7}{|c|}{ Cognitive capacity (NUCOG total score) } \\
\hline Initial $(n=86)$ & $90.06(7.0)$ & $87.7-92.4$ & $90.75(6.5)$ & $88.8-92.6$ & $90.02(6.7)$ & $89.0-91.9$ \\
\hline
\end{tabular}

Abbreviations: FIM, Functional Independence Measure; FSS, Fatigue Severity Scale.

${ }^{*} P<0.05$ between the two groups for HADS anxiety and SCS.

** $P<0.05$ over time for entire group.

the Australian community. ${ }^{6,18}$ Depression will be influenced by many factors, including pre-injury psychological status, severity of secondary conditions, cognitive impairment, and perceptions. ${ }^{6}$ Arguably, these factors will combine to determine how people with SCI adjust to the many challenges and difficulties experienced on a daily basis. ${ }^{19-21}$ The findings from our research suggest that this is especially true for those transitioning from the inpatient context (where they have frequent social and medical support) into the community where the person experiences a substantial reduction in support. This highlights the need for increased community resources to target individuals at risk of depression soon after they have transitioned into the community.

A major objective of this research was to investigate whether altering the dosage and style of delivery of CBT within SCI rehabilitation resulted in reduced depressive mood over time. One possible strategy was to increase exposure to a treatment like CBT and introduce group sessions within the rehabilitation phase. ${ }^{21}$ However, such a strategy was not found to be efficacious, given that increasing CBT dosage in the form of adding up to $12 \mathrm{~h}$ of group-CBT sessions integrated into SR added little or no value in terms of improving mood outcomes. This result is offset somewhat given the CBT group had twice the number with clinically elevated depressive mood at admission and close to double the rate at discharge compared with the SR group (see Table 4). Also, there was a small nonsignificant increase in depressive mood in the SR control at the 12-month post-injury measure, and this could be due to normal variation, or it could be related to a number of factors, such as these participants being less likely to seek psychological assistance during inpatient rehabilitation or increased stressors encountered when living in the community. The TOST test of equivalence also suggests that the two groups were not different in their mood associated with adding group-based CBT to rehabilitation. Future research should therefore investigate the potential of alternative strategies targeting depression, such as 


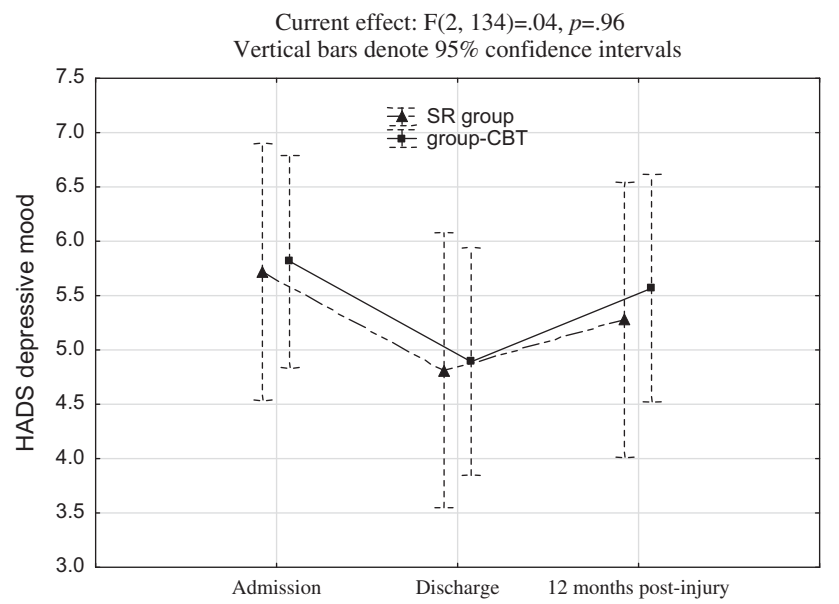

Figure 2 Change in depressive mood in those who received group-CBT versus those who received SR as participants transitioned from an inpatient hospital setting into the community. HADS anxiety and Secondary Conditions assessed at admission were entered as covariates in the analysis.

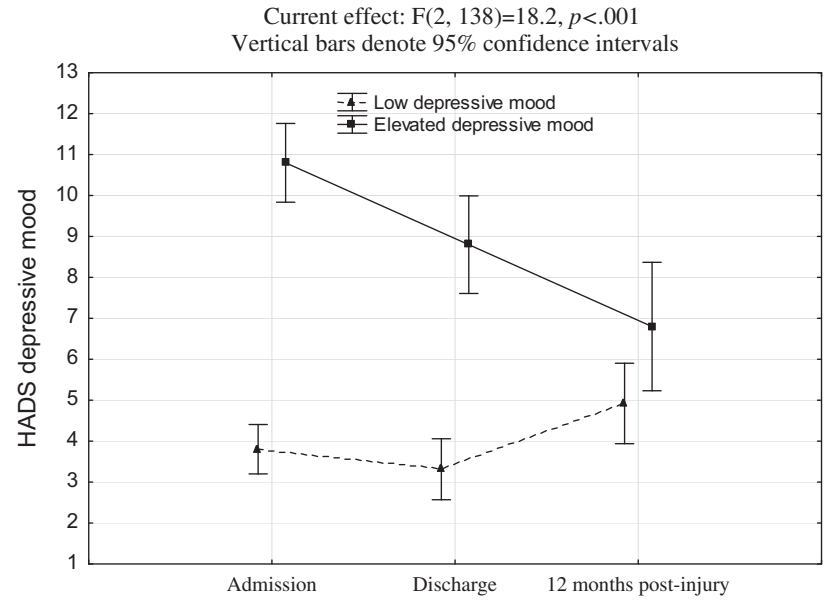

Figure 3 Change in depressive mood in those with clinically elevated depressive mood versus those with resilient mood (low depressive mood) as participants transitioned from an inpatient hospital setting into the community.

Table 3 Percentage of SR Control, Group-CBT and the total sample who had clinically elevated depressive mood (probable depression based on a HADS Depressive mood score of $\geqslant 8$ )

\begin{tabular}{llll}
\hline Time period & \multicolumn{1}{c}{ SR Control } & Group-CBT & Total sample \\
\hline Admission $(n=87)^{\mathrm{a}}$ & $15.7 \%(6 / 38 ; 7-30)$ & $30.6 \%(15 / 49 ; 19-44)$ & $24.1 \%(21 / 87 ; 16-34)$ \\
Discharge $(n=82)$ & $18.9 \%(7 / 37 ; 9-34)$ & $28.9 \%(13 / 45 ; 18-43)$ & $24.4 \%(20 / 82 ; 16-35)$ \\
12 months $(n=71)$ & $24.1 \%(7 / 29 ; 12-42)$ & $28.6 \%(12 / 42 ; 17-44)$ & $26.8 \%(19 / 71 ; 18-38)$ \\
\hline
\end{tabular}

Proportions and $95 \%$ confidence intervals are shown in brackets.

anote: Missing data for one participant.

employing sensitive algorithms based on inpatient data that can be used to predict mood outcomes in the community. 5,6 Other strategies include developing effective and innovative on-line interventions, ${ }^{49}$ especially those that are designed to enhance self-efficacy, given that low self-efficacy is related to higher risks of depression. ${ }^{50}$ Another strategy would be to determine individual preferences for treatment of depression during SCI rehabilitation. Fann et al. ${ }^{51}$ investigated treatment preferences for depression in 183 adult inpatients with SCI, of which $28 \%$ met criteria for probable major depression. They found physical exercise was the most preferred treatment option (preferred by almost $80 \%$ ), followed by antidepressant medication and individual counselling (both preferred by around 70\%). Of interest, group counselling was the least preferred option (preferred by 48\%). ${ }^{51}$ The Fann et al. study and our current findings suggest that it is crucial to determine preferred treatment options for depression in people during SCI rehabilitation. Finally, providing evidence-based education for inpatients on the most effective treatments for depression could also be an effective strategy if this does not already occur in SCI Units. For example, recommending a combination approach, such as improving lifestyle (e.g. increasing physical activity, improving diet and encouraging healthy sleep) combined with CBT and pharmacological therapies. ${ }^{52}$

Inspection of Figure 3 and the data in Table 4 indicates the majority remained resilient and stable over time, while a large minority were depressive. This supports findings of trajectory studies that indicate a majority of adults with acute SCI are resilient (stable or improving) over time, while a minority of up $30 \%$ are depressive (i.e. remaining depressive or deteriorating). ${ }^{19-21}$ Similar mental health trajectories were also found in eight Dutch rehabilitation centres with specialized SCI units. ${ }^{53}$ Notably, in this current research, the depressive group experienced a significant reduction in depressive mood after their transition into the community, compared with depressive mood assessed during rehabilitation. Despite this improvement, depressive mood still remained at the top of the normal mood range, raising concerns that mood may deteriorate over the longer term.

The above findings suggest mental health resources should be strategically concentrated on those assessed with elevated depressive mood during their rehabilitation phase. Further research could investigate the benefits of targeting those at risk of depression and providing preference of treatment such as individual or group-CBT. Chronic pain is usually positively associated with depressive mood, ${ }^{2,3}$ and the finding that pain intensity scores had significantly increased 6 months after discharge also provides direction for improving outcomes; for example, promoting the importance of instructing persons with SCI in pain self-management skills before they are discharged. ${ }^{12,54}$

Findings confirmed the lack of significant association between sociodemographic and injury-related variables and depressive mood outcomes. ${ }^{6}$ Weak negative associations were found between depressive mood outcomes and years of education and cognitive capacity, reinforcing the important role these factors play in adjustment. Depressive mood assessed at admission and discharge was significantly associated with depressive mood, fatigue and pain catastrophizing 12 months post-injury. This suggests that to improve depression outcomes it may be necessary to target additional factors such as fatigue and pain catastrophizing. ${ }^{4,5}$ 
Table 4 Depressive mood scores for the resilient subgroup $(n=51)$ and those with clinically elevated depressive mood $(n=20)$ over time (based on a HADS depressive mood cutoff of $\geqslant 8$ )

\begin{tabular}{|c|c|c|c|}
\hline \multirow{2}{*}{ Subgroup } & Admission & Discharge & 12 months post-injury \\
\hline & Mean (s.d.) $95 \% \mathrm{Cl}$ & Mean (s.d.) $95 \% \mathrm{Cl}$ & Mean (s.d.) 95\% Cl \\
\hline Resilient mood & 3.8 (2) $3.3-4.3$ & 3.5 (3) $2.7-4.2$ & 4.9 (3) 3.9-5.9 \\
\hline Clinically elevated depressive mood & 10.8 (2) 9.9-11.8 & $8.8(2) 7.7-9.9$ & 6.8 (3) $5.2-8.4$ \\
\hline
\end{tabular}

$95 \% \mathrm{Cl}, 95 \%$ confidence intervals around the mean.

Table 5 Antidepressant medication as a percentage of the total sample as a function of clinically elevated depressive mood at 12 months post-injury

\begin{tabular}{lcc}
\hline Medication & \multicolumn{2}{c}{ Elevated depressive mood $($ HADS $\geqslant 8)$} \\
\cline { 2 - 3 } & No & Yes \\
\hline No & $53.6(37 / 69)$ & $20.3(14 / 69)$ \\
Yes & $20.3(14 / 69)$ & $5.8(4 / 69)$ \\
\hline
\end{tabular}

Note: medication data missing for two participants, therefore $n=69$.

Proportions of the total sample are provided in brackets.

Table 6 Pearson correlation coefficients between depressive mood at the three time periods and psychosocial factors at admission, discharge and 12-month post-injury for the total group

\begin{tabular}{|c|c|c|c|}
\hline \multirow[t]{3}{*}{ Variables } & \multicolumn{3}{|c|}{ HADS depressive mood score } \\
\hline & Admission & Discharge & 12 months \\
\hline & $\mathrm{n}=88$ & $\mathrm{n}=82$ & $\mathrm{n}=71$ \\
\hline \multicolumn{4}{|l|}{ Anxiety } \\
\hline Admission & $0.60 * * *$ & $0.42 * * *$ & $0.33 * *$ \\
\hline Discharge & $0.50 * * *$ & $0.65^{* * *}$ & $0.38 * *$ \\
\hline 6 months & $0.37 * *$ & $0.45^{* * *}$ & $0.76^{* * *}$ \\
\hline \multicolumn{4}{|l|}{ Fatigue } \\
\hline Admission & $0.39 * *$ & 0.14 & 0.06 \\
\hline Discharge & $0.45^{* *}$ & $0.44^{* *}$ & 0.20 \\
\hline 6 months & 0.00 & 0.13 & $0.45^{* *}$ \\
\hline \multicolumn{4}{|l|}{ Pain intensity } \\
\hline Admission & 0.12 & 0.11 & -0.09 \\
\hline Discharge & 0.22 & $0.30^{*}$ & 0.10 \\
\hline 6 months & 0.12 & 0.12 & 0.25 \\
\hline \multicolumn{4}{|c|}{ Pain catastrophization } \\
\hline Admission & $0.35^{*}$ & $0.43^{* *}$ & 0.09 \\
\hline Discharge & $0.30 *$ & $0.43^{* *}$ & 0.11 \\
\hline 6 months & 0.19 & 0.17 & $0.47^{* *}$ \\
\hline \multicolumn{4}{|c|}{ Severity of secondary conditions } \\
\hline Admission & 0.22 & 0.04 & $0.29 *$ \\
\hline Discharge & $0.25^{*}$ & 0.22 & $0.32 * *$ \\
\hline 6 months & 0.14 & 0.03 & $0.42 * *$ \\
\hline
\end{tabular}

Note: ${ }^{*} P<0.05 ;{ }^{*} P<0.01 ;{ }^{* *} P<0.001$
Limitations require discussion. Given SCI rehabilitation is composed of multiple treatment components and driven by a multidisciplinary team, it is a challenge to determine the efficacy of any one component for improving mood. Future research needs to investigate which specific components of rehabilitation are efficacious in the treatment of depressive mood following SCI. Conducting the study over a small number of SCI Units $(n=3)$ was a limitation, given the limited numbers of participants sustaining an acute SCI in any 1 year discouraged the employment of an randomized control trial involving group-CBT. A preferable approach would have been to employ a cluster randomized controlled trial design in which SCI Units around Australia were randomized to either SR or the addition of group-CBT. Additional limitations include a recruitment rate of just over $60 \%$, and assessing outcomes only 12 months post-injury. The collection of longer-term mood data is desirable, with 3-5 years postdischarge outcomes currently being conducted. While the significant reduction in depressive mood seen in those with clinically elevated depressive mood could be interpreted as regression to the mean, such an interpretation is not supported by trajectory research or that depression is a long-term problem for a large minority. ${ }^{19-21,53}$ Further, it is possible that the drop-out rate influenced mood differences between the CBT and SR groups at the 12-month post-injury assessment, and differences in depressive mood between the CBT and SR groups could also have been influenced by the greater number of females in the CBT group, given females have higher risk of depression. ${ }^{55}$ However, a Rasch analysis conducted on the HADS showed gender bias was minimal when responding to the HADS mood items. ${ }^{56}$ The antidepressant medication usage in the different groups (e.g. shown in Table 5) is also a possible limitation. The data in Table 5, for example, suggest those using antidepressants were more likely to have HADS mood scores $<8$. Therefore, depressive mood results have likely been influenced by whether participants were prescribed medications for their mood.

Future research is needed that examines individual differences related to common trajectories of how people adjust following SCI and which controls for many of the potential limitations discussed. In conclusion, it is hoped that this research will contribute to lowering the substantial rates of depression in people with SCI as they transition into the community.

\section{DATA ARCHIVING}

There were no data to deposit.

\section{CONFLICT OF INTEREST}

The authors declare no conflict of interest.

\section{ACKNOWLEDGEMENTS}

This work was financially supported by the Lifetime Care Support Authority, NSW, Australia (LTCSA Ref 08/209). We thank the following for assisting in the 
study: Ms Annalisa Dezarnaulds, Ms Alison Hales, Ms Catherine Ephraums, and Associate Professor Kathryn Nicholson Perry. We would also like to acknowledge the tremendous work that Professor Paul Kennedy has contributed to research designed to improve the life of people with SCI. He will be greatly missed.

1 Somers MF. Spinal Cord Injury: Functional Rehabilitation. Chicago: Prentice Hall, 2001.

2 Jensen MP, Kuehn CM, Amtmann D, Cardenas DD. Symptom burden in persons with spinal cord injury. Arch Phys Med Rehabil 2007; 88: 638-645.

3 Tate DG, Forchheimer MB, Karana-Zebari D, Chiodo AE, Thomas JYK. Depression and pain among inpatients with spinal cord injury and spinal cord disease: differences in symptoms and neurological function. Disabil Rehabil 2013; 35: 1204-1212.

4 Wijesuriya N, Tran Y, Middleton J, Craig A. The impact of fatigue on the health related quality of life in persons with spinal cord injury. Arch Phys Med Rehabil 2012; 93: 319-324.

5 Craig A, Rodrigues D, Tran Y, Guest R, Bartrop R, Middleton J. Developing an algorithm capable of discriminating depressed mood in people with spinal cord injury. Spinal Cord 2014; 52: 413-416.

6 Craig A, Nicholson Perry K, Guest R, Tran Y, Dezarnaulds A, Hales A et al. A prospective study of the occurrence of psychological disorders and co-morbidities following spinal cord injury. Arch Phys Med Rehabil 2015; 96: 1426-1434.

7 Fann JR, Bombardier CH, Richards JS, Tate DG, Wilson CS, Temkin N. Depression after spinal cord injury: comorbidities, mental health service use, and adequacy of treatment. Arch Phys Med Rehabil 2011; 92: 352-360.

8 Hancock K, Craig AR, Tennant C, Chang E. The influence of spinal cord injury on coping styles and self-perceptions: a controlled study. Aust N Z J Psychiatry 1993; 27: $450-456$.

9 Hoffman JM, Bombardier CH, Graves DE, Kalpakjian CZ, Krause JS. A Iongitudinal study of depression from 1 to 5 years after spinal cord injury. Arch Phys Med Rehabil 2011; 92: 411-418.

10 Kennedy P, Rogers BA. Anxiety and depression after spinal cord injury: a longitudinal analysis. Arch Phys Med Rehabil 2000; 81: 932-937.

11 Williams R, Murray A. Prevalence of depression after spinal cord injury: a meta-analysis. Arch Phys Med Rehabil 2015; 96: 133-140.

12 Krueger H, Noonan VK, Williams D, Trenaman LM, Rivers CS. The influence of depression on physical complications in spinal cord injury: behavioral mechanisms and health-care implications. Spinal Cord 2013; 51: 260-266.

13 Soden R, Walsh J, Middleton J, Craven M, Rutkowski S, Yeo J. Causes of death after spinal cord injury. Spinal Cord 2000; 38: 604-610.

14 Tate D, Forchheimer M, Maynard F, Dijkers M. Predicting depression and psychological distress in persons with spinal cord injury based on indicators of handicap. Am J Phys Med Rehabil 1994; 73: 175-183.

15 Craig A, Tran Y, Middleton J. Psychological morbidity and spinal cord injury: a systematic review. Spinal Cord 2009; 47: 108-114.

16 Kishi Y, Robinson RG, Forrester AW. Prospective longitudinal study of depression following spinal cord injury. J Neuropsychiatry Clin Neurosci 1994; 6: 237-244.

17 Craig AR, Hancock KM, Dickson HG. A longitudinal investigation into anxiety and depression in the first 2 years following a spinal cord injury. Paraplegia 1994; 32: 675-679.

18 Andrews G, Henderson S, Hall W. Prevalence, comorbidity, disability and service utilisation: overview of the Australian National Mental Health Survey. Br J Psychiatry 2001; 178: 145-153.

19 Bonanno GA, Kennedy P, Galatzer-Levy IR, Lude P, Elfström ML. Trajectories of resilience, depression, and anxiety following spinal cord injury. Rehabil Psychol 2012; 57: 236-247.

20 Bombardier CH, Adams LM, Fann JR, Hoffman JM. Depression trajectories during the first year after spinal cord injury. Arch Phys Med Rehabil 2016; 97: 196-203.

21 Guest R, Craig A, Nicholson Perry K, Tran Y, Hales A, Ephraums C et al. Resilience following spinal cord injury: a prospective controlled study investigating the influence of the provision of group CBT during inpatient rehabilitation. Rehabil Psychol 2015; 60: 311-321.

22 Middleton J, Craig A. Psychological challenges in treating persons with spinal cord injury. In: Craig A, Tran Y (eds). Psychological Dynamics Associated with Spinal Cord Injury Rehabilitation: New Directions and Best Evidence, Chapter 1. Nova Science Publishers: New York, 2008, pp 3-54.

23 Fann JR, Bombardier CH, Richards JS, Wilson CS, Heinemann AW, Warren AM et al. Venlafaxine extended-release for depression following spinal cord injury: a randomized clinical trial. JAMA Psychiatry 2015; 72: 247-258.

24 Elliott TR, Kennedy P. Treatment of depression following spinal cord injury: an evidence-based review. Rehabil Psychol 2004; 49: 134-139.

25 Mehta S, Orenczuk S, Hansen KT, Aubut JA, Hitzig SL, Legassic M et al. An evidencebased review of the effectiveness of cognitive behavioral therapy for psychosocial issues post-spinal cord injury. Rehabil Psychol 2011; 56: 15-25.
26 Post M, Van Leeuwen C. Psychosocial issues in spinal cord injury: a review. Spinal Cord 2012; 50: 382-389.

27 Dorstyn D, Mathias J, Denson L. Efficacy of cognitive behavior therapy for the management of psychological outcomes following spinal cord injury: a meta-analysis. J Health Psychol 2011; 16: 374-391.

28 Dorstyn DS, Mathias JL, Denson LA. Psychological intervention during spinal rehabilitation: a preliminary study. Spinal Cord 2010; 48: 756-761.

29 Kennedy P, Duff J, Evans M, Beedie A. Coping effectiveness training reduces depression and anxiety following traumatic spinal cord injuries. $\mathrm{Br} J \mathrm{Clin}$ Psychol 2003; 42: 41-52.

30 Duchnick JJ, Letsch EA, Curtiss G. Coping effectiveness training during acute rehabilitation of spinal cord injury/dysfunction: a randomized clinical trial. Rehabil Psychol 2009; 54: 123-132.

31 Craig A, Hancock K, Chang E, Dickson H. Immunizing against depression and anxiety after spinal cord injury. Arch Phys Med Rehabil 1998; 79: 375-377.

32 Craig A. In: Craig A, Tran Y (eds). Psychological Dynamics Associated with Spinal Cord Injury Rehabilitation: New Directions and Best Evidence, Chapter 5. Nova Science Publishers: New York, 2008, pp 131-154.

33 De Vivo MJ, Richards JS, Stover SL, Go BK. Spinal cord injury. Rehabilitation adds life to years. West J Med 1991; 154: 602-606.

34 Craig A, Moses P, Tran Y, Mclsaac P, Kirkup L. The effectiveness of a "hands free" environmental control system for the profoundly disabled. Arch Phys Med Rehabil 2002; 83: 1455-1458.

35 Bonell CP, Hargreaves J, Cousens S, Ross D, Hayes R, Petticrew M et al. Alternatives to randomisation in the evaluation of public health interventions: design challenges and solutions. J Epidemiol Community Health 2011; 65: 582-587.

36 Lammertse D, Tuszynski M, Steeves J, Curt A, Fawcett J, Rask C et al. Guidelines for the conduct of clinical trials for spinal cord injury as developed by the ICCP panel: clinical trial design. Spinal Cord 2007; 45: 232-242.

37 Cripps RA, Harrison JE. Injury as a Chronic Health Issue in Australia. Australian Institute Health Welfare: Canberra, 2008.

38 Woolrich RA, Kennedy P, Tasiemski T. A preliminary psychometric evaluation of the Hospital Anxiety and Depression Scale (HADS) in 963 people living with a spinal cord injury. Psychol Health Med 2006; 11: 80-90.

39 Krupp LB, LaRocca NG, Muir-Nash J, Steinberg AD. The Fatigue Severity Scale; application to patients with multiple sclerosis and systemic lupus erythematosus. Arch Neurol 1989; 46: 1121-1123.

40 Jensen MP, Turner JA, Romano JM, Fisher LD. Comparative reliability and validity of chronic pain intensity measures. Pain 1999; 83: 157-162.

41 Flor $\mathrm{H}$, Behle DJ, Birbaumer N. Assessment of pain-related cognitions in chronic pain patients. Behav Res Ther 1993; 31: 63-73.

42 Walterfang M, Siu R, Velakoulis D. The NUCOG: validity and reliability of a brief cognitive screening tool in neuropsychiatric patients. Aust N Z J Psychiatry 2006; 40: 995-1002.

43 Hall KM, Cohen ME, Wright J, Call M, Werner P. Characteristics of the Functional Independence Measure in traumatic spinal cord injury. Arch Phys Med Rehabil 1999; 80: 1471-1476.

44 Kalpakjian CZ, Scelza WM, Forchheimer MB, Toussaint LL. Preliminary reliability and validity of a spinal cord injury Secondary Conditions Scale. J Spinal Cord Med 2007; 30: $131-139$

45 Faul FG. *Power Version 3.1.9.2. Kiel University: Germany, 2014

46 Walker E, Nowacki AS. Understanding equivalence and non-inferiority testing. J Gen Intern Med 2011; 26: 192-196.

47 Jaccard J, Guilamo-Ramos V, Johansson M, Bouris A. Multiple regression analyses in clinical child and adolescent psychology. J Clin Child Adolesc Psychol 2006; 35: 456-479.

48 Cohen J. Statistical Power Analysis for the Behavioral Sciences. New Jersey: LEA, 1988.

49 Verwer JH, van Leeuwen CM, Bolier L, Post MW. Feasibility of an online well-being intervention for people with spinal cord injury: a pilot study. Spinal Cord 2016; 54: 473-477.

50 Munce SE, Straus SE, Fehlings MG, Voth J, Nugaeva N, Jang E et al. Impact of psychological characteristics in self-management in individuals with traumatic spinal cord injury. Spinal Cord 2016; 54: 29-33.

51 Fann JR, Crane DA, Graves DE, Kalpakjian CZ, Tate DG, Bombardier CH. Depression treatment preferences after acute traumatic spinal cord injury. Arch Phys Med Rehabil 2013; 94: 2389-2395.

52 Malhi GS, Bassett D, Boyce P, Bryant R, Fitzgerald PB et al. Royal Australian and New Zealand College of Psychiatrists clinical practice guidelines for mood disorders. Aust N Z J Psychiatry 2015; 49: 1087-1206.

53 van Leeuwen CM, Hoekstra T, van Koppenhagen CF, de Groot S, Post MW. Trajectories and predictors of the course of mental health after spinal cord injury. Arch Phys Med Rehabil 2012; 93: 2170-2176.

54 Craig A, Tran Y, Siddall P, Wijesuriya N, Lovas J, Bartrop R et al. Developing a model of associations between chronic pain, depressive mood, chronic fatigue and self-efficacy in people with spinal cord injury. J Pain 2013; 14: 911-920.

55 Piccinelli M, Wilkinson G. Gender differences in depression. Br J Psychiatry 2000; 177: 486-492.

56 Müller R, Cieza A, Geyh S. Rasch analysis of the Hospital Anxiety and Depression Scale in spinal cord injury. Rehabil Psychol 2012; 57: 214-223. 